FACTA UNIVERSITATIS

Series: Mechanical Engineering Vol. 16, N 3, 2018, pp. 389 - 403

https://doi.org/10.22190/FUME171121020U

Original scientific paper

\title{
SMART EQUIPMENT DESIGN CHALLENGES FOR REAL-TIME FEEDBACK SUPPORT IN SPORT
}

UDC 528.835:796.012

\section{Anton Umek, Anton Kos}

Faculty of Electrical Engineering, University of Ljubljana, Slovenia

\begin{abstract}
Smart equipment can support feedback in motor learning process. Smart equipment with integrated sensors can be used as a standalone system or complemented with body-attached wearable sensors. Our work focuses on real-time biofeedback system design, particularly on the application of a specific sensor selection. The main goal of our research is to prepare the technical conditions to prove efficiency and benefits of the realtime biofeedback when used in selected motion-learning processes. The most used wireless technologies that are used or are expected to be used in real-time biofeedback systems are listed. The tests performed on two prototypes, smart golf club and smart ski, show an appropriate sensor selection and feasibility of implementation of the real-time biofeedback concept in golf and skiing practice. We are confident that the concept can be expanded for use in other sports and rehabilitation. It has been learned that at this time none of the existing wireless technologies can satisfy all possible demands of different real-time biofeedback applications in sport.
\end{abstract}

Key Words: Smart Equipment, Motor Learning, Sport, Biofeedback Systems, Sensors, Actuators, Wireless Networks

\section{INTRODUCTION}

Feedback is the most important concept for learning except practice itself [1]. During the practice, the natural (inherent) feedback information is provided internally through human sense organs. Augmented feedback is provided by an external source, traditionally by instructors and trainers [2]. Modern technical equipment can help both the performer and the instructor. In many sports disciplines video recording is a classical method for providing additional feedback information for post analysis and terminal feedback. Modern technical equipment can provide more precise measurements of human kinetics

Received November 21, 2017 / Accepted May 15, 2018

Corresponding author: Anton Kos

Faculty of Electrical Engineering, University of Ljubljana, Tržaška c. 25, 1000 Ljubljana, Slovenia

E-mail: anton.kos@fe.uni-lj.si 
and kinematics parameters and can considerably improve the quality of feedback information. Modern optical motion tracking systems are using passive or active markers and a number of high-speed cameras. An alternative to optical tracking systems are inertial motion unit (IMU) based systems, which use several wearable sensors attached to the human body. Both types of motion tracking systems are professional and expensive equipment that can be used not only for biomechanics research in sports, rehabilitation and ergonomics but also as an animation tool in movie industry and virtual reality tracking. Augmented feedback supported by technical equipment (sensors and actuators) is defined as biofeedback because a human is inside the feedback loop. The general architecture of a biofeedback system is presented in Fig. 1.

In a biofeedback system, a person has attached sensors that measure body functions and actions. The sensors are connected to a processing device for sensor signal and data analysis. The results are communicated back to the person through one of the human senses. The person attempts to act on the received information to change the body function or action in the desired manner. The term biofeedback was first described in connection with the human physiological processes and shortly afterwards in terms of the physical body activity in sports biomechanics. According to [3], biofeedback can be categorized into two main groups: physiological and biomechanical. In this paper, the word biofeedback concerns body and with body-related activity in the sense of physical movement; it is classified as biomechanical biofeedback in [3].

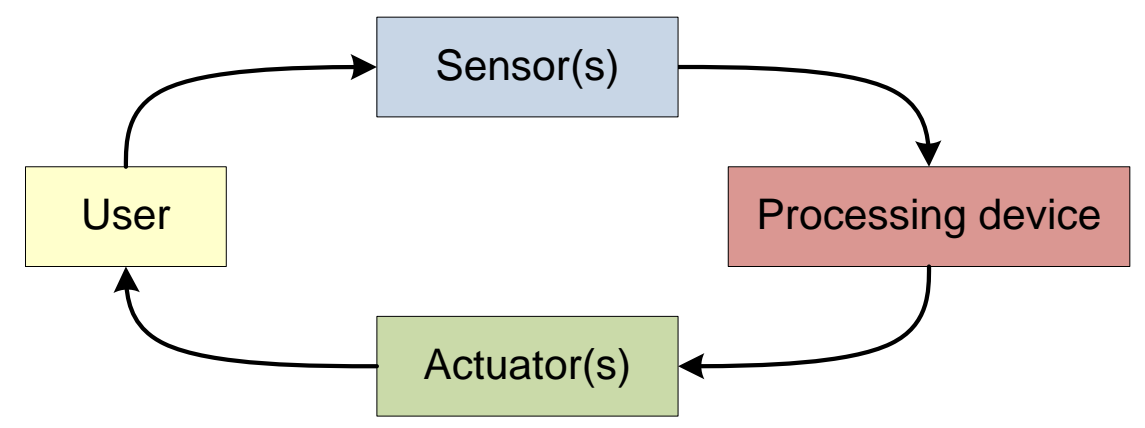

Fig. 1 General architecture of a biofeedback system

To achieve a widespread use of biofeedback applications, important feedback information concerning knowledge of performance should be provided with less complex and cheaper technical equipment. Miniature IMU sensors (accelerometers and gyroscopes) are integrated in every modern smartphone. Consequently, many motion activity applications for smartphones and wearables using only accelerometer data exist.

In many sport disciplines various types of equipment are essential or even indispensable for performing the desired task (tennis rackets, baseball bats, golf clubs, alpine skis, etc.). In fact, some of the most relevant human actions are transferred through the equipment. All this equipment can be supported by different types of sensors, not only accelerometers, gyroscopes and magnetometers. For example, strain sensors are the perfect choice to detect and measure force, torque, and bending in different parts of the equipment. An appropriate sensor fusion algorithm can give precise information on performers' actions and equipment reactions. A 
feedback from the sport equipment could therefore improve the performer's skills, especially if it is provided in real-time, that is, without a significant delay. Smart sport equipment can include any combination of sensor(s), processing device, and actuators(s) as defined in Fig. 1.

Sport equipment manufacturers have already started embedding the digital technology into their products. Some examples of smart sport equipment, which are already available on the global market, are: smart shoes, smart tennis racket, smart basketball, smart baseball bat, and smart golf club [4]. In the near future it is possible to predict many improvements in technology that could drive down the prices and encourage a widespread adoption of smart sport equipment. The real-world application of smart sport equipment as a part of biofeedback systems face several constraints that can represent higher or lower obstacles in their acceptance and use.

The space constraint defines the possible locations of biofeedback system elements: (a) personal space system, where all system elements are attached to the user, (b) confined space system, where the elements are distributed within a defined and limited space, and (c) open space system, where elements are not restricted in space.

The time constraint defines the timing of the feedback given by the biofeedback system, which can work only if the feedback loop is closed. That means that the user receives, understands, and possibly reacts to the feedback information. The feedback information can be given at different times: (a) terminal feedback is given after the activity has been performed; (b) concurrent feedback is given during the activity. Feedback loop delay consists of communication delays for the transmission of sensor and feedback signals, processing delay, and user reaction delay.

Computation constraint is closely related and dependent on the space and time constraints as well as on the properties of sensors and actuators. Processing in the biofeedback loop can be done in real time or in post processing. While the post processing mode does not represent a computational problem to the most of the processing devices and communication technologies, real time operation can many times be a difficult problem because the processing device has to finish the processing within the time frame of one sensor sampling period, which can be as low as $1 \mathrm{~ms}$ or even less.

Another important parameter is the communication delay within the biofeedback loop. This parameter is connected to all of the constraints studied above. Communication delay is heavily dependent on the communication technology used. For the real-time biofeedback systems the communication delay must be a fraction of the reaction delay.

\section{MotiVATION}

In majority of research work in sport wearable sensors are used for the purpose of monitoring and for the post processing analysis of signals and data. The feedback information is given with delay after the performed activity, what is defined as terminal feedback. The same is true for the majority of sport applications already available on smartphones; post processing with presentation of some vital or important parameters. The concurrent feedback, which is given in real time within the currently performed action, is very useful for motor learning, but is rarely used.

The primary aim of our research is the development of technical equipment that would allow implementation of real-time biomechanical biofeedback systems. We are convinced 
that such systems would allow a leap in research in this field. The important tasks in our research are the selection of appropriate sensors and the assurance of suitable conditions for sensor signal transmission and processing. As it is evident from research papers, IMU sensors are the most often used ones in sports [5-12]. One promising direction of research is the use of smartphones in place of one or several elements of the biofeedback loop. Smartphones can bring many important advantages, especially in bringing biofeedback systems closer to amateur users, who cannot afford expensive expert systems. Smartphone properties and their suitability for biofeedback applications have been studied in [13-17].

The main focus of our current research is motor learning in sport with the help of feedback information provided by sensors integrated into sport equipment $[18,19]$. The results of our research can be implemented also in the field of rehabilitation equipment as motor learning in rehabilitation is generally less demanding from that in sport. Research efforts in smart sport equipment, many times coupled with sensors attached to the athlete, are present in many sports: from swimming [20], rowing [21, 22], kayak [23], canoe [24], and precision shooting [25], to golf and skiing that are presented and discussed later in this paper.

One of the main research motivations of this paper is also the identification and selection of the most appropriate wireless communication technologies for various biofeedback applications that include smart sport equipment. To date, there is no one-fitsall solution to the above challenge. The sources of the data are sensors that are very heterogeneous in many aspects. They produce data rates from of a few bytes per minute for measuring patient's temperature to a few Mbit/s for a high-resolution and high-speed camera used in sport. Sensors can be used for measuring physiological processes of a human (heart rate, glucose levels, blood saturation, etc.) to measuring performance of an athlete or sport equipment (high dynamic movement, high frequency vibrations, bending, strain, etc.). The same heterogeneity is expressed in the variety of sensor network technologies; from technologies that cover body area (BAN) to technologies that cover metropolitan area (MAN), from technologies with bitrates of a few kbit/s to technologies of a few hundreds of Mbit/s, from frequencies of $400 \mathrm{MHZ}$ to frequencies of $60 \mathrm{GHz}$, etc. It is obvious that the heterogeneity of sensors, networking technologies and application demands will yield the heterogeneity of the most appropriate solutions.

We would like to emphasize that this paper represents an interdisciplinary research in the fields of communication technologies, sensors, feedback systems, and sports. During our study and experiments in the abovementioned fields we have come across important obstacles and challenges that have not yet been addressed properly.

The main contributions of our paper are: (a) a systematic approach to the design of smart equipment that acts as a component of feedback systems in sport, (b) setting a guideline for the selection of the most appropriate combination of sensors, actuators and wireless technologies for different variants of real-time feedback systems in sport. To the best of our knowledge, there are no research papers that jointly discuss the topics in this paper. 


\section{SMART SPORT EQUiPMENT SENSORS, ACTUATORS AND WIRELESS TECHNOLOGIES}

The integration of sensors and actuators into sport equipment enables not only the acquisition of information about motion, static positions, and acting forces, but also the means of giving appropriate feedback information back to the user. Using the sensor signal analysis and specific a priori knowledge, the validation of the movement correctness can be achieved and appropriate feedback information can be communicated within the biofeedback loop. This procedure was tested in practical experiments in the field of training in golf and alpine skiing that is presented in Section 4.

\subsection{Sensors and actuators}

Sensors and actuators used in sports are heterogeneous in their properties. They can be grouped based on different criteria, such as measured quantity, bit rate, sampling rate, accuracy, precision, and similar [26-28]. Some of the most popular sensors and actuators used in sport are presented in Table 1.

Table 1 Sensors and actuators used in sport

\begin{tabular}{lcc}
\hline Sensor/Actuator & Bit rate & Delay \\
\hline Temperature & $<100 \mathrm{bit} / \mathrm{s}$ & Not critical \\
Heart rate & $<100 \mathrm{bit} / \mathrm{s}$ & Seconds \\
ECG & $20-100 \mathrm{kbit} / \mathrm{s}$ & $<1 \mathrm{~s}$ \\
Accelerometer & $1-200 \mathrm{kbit} / \mathrm{s}$ & $<50 \mathrm{~ms}$ \\
Gyroscope & $1-200 \mathrm{kbit} / \mathrm{s}$ & $<50 \mathrm{~ms}$ \\
Strain-gauge & $1-50 \mathrm{kbit} / \mathrm{s}$ & $<50 \mathrm{~ms}$ \\
Tactile actuator & $<100 \mathrm{bit} / \mathrm{s}$ & $<50 \mathrm{~ms}$ \\
Audio actuator & $<1 \mathrm{Mbit} / \mathrm{s}$ & $<50 \mathrm{~ms}$ \\
Video actuator & $<10 \mathrm{Mbit} / \mathrm{s}$ & $<50 \mathrm{~ms}$ \\
\hline
\end{tabular}

Several sensor groups can be distinguished: (a) Sensors for low or high dynamic physiological processes, (b) sensors for low dynamic movement activities and (c) sensors for high dynamic movement activities. The main parameters that correspond to abovementioned groups are: sampling frequency (from below $1 \mathrm{~Hz}$ to a few $\mathrm{kHz}$ ), precision (from 8 to $16 \mathrm{bit}$ ), and produced bit rate (from less than $1 \mathrm{bit} / \mathrm{s}$ to tens of Mbit/s). Actuators show less variety in their type and output, but can vary as much as sensors in sampling frequency and bit rate; from a one-bit tactile actuator (buzzer) to a high-definition and high-speed video screen. By combining several sensors and actuators within one (real-time) biofeedback system, the required bit rates and delay constraints can be very high. The selection of the most appropriate wireless technology is of paramount importance for achieving high quality of service of biofeedback system operation.

\subsection{Wireless technologies}

Like sensors and actuators, wireless technologies are also very heterogeneous in their properties. Table 2 lists the most used wireless communication technologies that are in use today for providing the functionalities of BAN, PAN (Personal Area Network), LAN (Local Area Network), and MAN. Only the range and bit rate properties that are the most 
important for our discussion are listed. More details about the listed technologies can be found in [26]. The selection of the most appropriate wireless communication technology depends on the type and implementation of the biofeedback systems. The heterogeneity of wireless technologies and the variety of biofeedback system versions suggests the use of multi-radio concepts [26-28].

Table 2 Standardized wireless technologies with potential use in sport

\begin{tabular}{lcc}
\hline Technology & Range & Bit rate \\
\hline Bluetooth & $10-100 \mathrm{~m}$ & $1-3 \mathrm{Mbit} / \mathrm{s}$ \\
ZigBee & $10-100 \mathrm{~m}$ & $20-250 \mathrm{kbit} / \mathrm{s}$ \\
IEEE 802.11n & $70 \mathrm{~m}$ & $600 \mathrm{Mbit} / \mathrm{s}$ \\
IEEE 802.11ac & $35 \mathrm{~m}$ & $6.93 \mathrm{Gbit} / \mathrm{s}$ \\
IEEE 802.11ah & $1 \mathrm{~km}$ & $40 \mathrm{Mbit} / \mathrm{s}$ \\
IEEE 802.11af & $>1 \mathrm{~km}$ & $1.8-26.7 \mathrm{Mbit} / \mathrm{s}$ \\
LoRaWAN & to $100 \mathrm{~km}$ & $250-5470 \mathrm{bit} / \mathrm{s}$ \\
\hline
\end{tabular}

\subsection{Selection of technologies for real-time feedback system}

A large number of factors and constraints have to be considered when selecting the most appropriate elements of feedback systems in sport. This is particularly true for the feedback systems that operate in real time (time constraint) giving concurrent feedback to the user. For help with the selection process, we have composed Fig. 2 that illustrates the available bitrates of wireless technologies from Table 2 plotted against their ranges, bitrate ranges of various sensors and actuators from Table 1, and space constraints of feedback systems defined in the Introduction. Computational constraint is not addressed in detail here because it can be controlled to a high degree by the system designer while the other two constraints are mostly the given properties of the feedback system. It should be noted that IEEE 802.11ah, IEEE 802.11af wireless technologies are trying to fill-in the gap for open space systems with kilometre ranges and bitrates in Mbit/s, but at the time of writing they were not yet available in the market.

A number of important conclusions for the design of real-time feedback system can be drawn from Fig. 2. For example: (a) real-time feedback systems based on physiological parameters, such as temperature and heart rate, can be implemented in personal, confined, and open space by using LoRaWAN wireless technology; (b) usage of audio and video actuators in personal space systems is supported by Bluetooth, IEEE 802.11n, IEEE 802.11ac, IEEE 802.11ah, and IEEE 802.11af wireless technologies; in open space systems these actuators can be used to some extent by implementing IEEE 802.11ah, IEEE 802.11af wireless technologies; the problem is that the latter two technologies are standardized, but not yet available in the market; (c) inertial sensors (accelerometer and gyroscope) can be used in personal and confined space systems by using all listed wireless technologies, except LoRaWAN, but that is always true only for one such sensor; if there are more inertial sensors in the feedback system, some of the above technologies can prove insufficient; (d) given the ZigBee based system in confined space, sensors for low dynamic physiological processes and a limited number of inertial and strain-gauge sensors with low sample rates can be used. Many similar useful conclusions can be made based on information included in Fig. 2. 


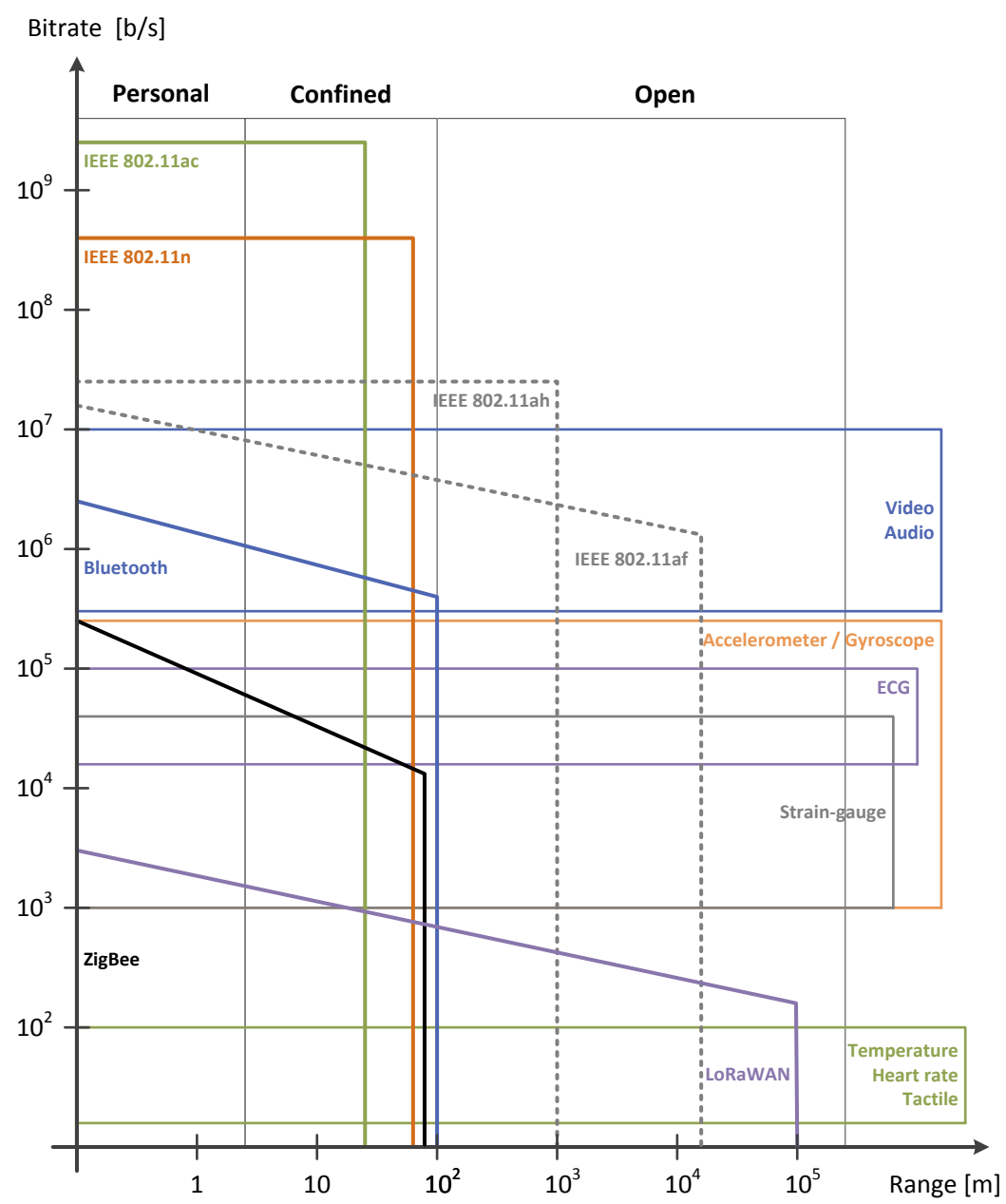

Fig. 2 Illustration of available bitrates of wireless technologies from Table 2 plotted against their ranges, bitrate ranges of various sensors and actuators from Table 1, and space constraints help with the selection of appropriate feedback system elements

\section{SMART SPORT EQUIPMENT PROTOTYPES}

For the validation of the above presented concept, two original smart equipment prototypes have been developed: smart golf club and smart ski. The development of realtime biofeedback system usage concepts and procedures were started based on the first measurement results. They include a systematic approach by defining the useful short practice lessons, adapting the precision of real-time biofeedback system to the capabilities of the user (amateur vs. professional), the choice of correct feedback modality, and the appropriate amount of feedback information adapted to the limited perception capabilities of users during training. 


\subsection{Smart golf club}

The smart golf club prototype includes: (a) two strain gage sensors, which measure the golf club shaft bend and (b) 3-axis MEMS accelerometer and 3-axis MEMS gyroscope, which measure acceleration and angular speed of the golf club. The latter two sensors are a part of the independent Shimmer 3 IMU equipped with Bluetooth communication interface. Strain gage sensor signals are acquired by the professional measurement system (National Instruments Corporation, Austin, TX, USA) with NI cRIO 9063 base (667 MHz dual-core controller with FPGA) with bridge amplifier module NI 9237. IMU sensor signals are acquired by the LabVIEW ${ }^{\mathrm{TM}}$ application running on the laptop.

Shimmer 3 devices can reliably stream sensor data using the Bluetooth up to sampling frequencies of $512 \mathrm{~Hz}$, which was used in our experiments. The accelerometer's dynamic range is up to $\pm 16 \mathrm{~g} 0$ and the gyroscopes dynamic range is up to $\pm 2000 \mathrm{deg} / \mathrm{s}$. The precision of both is 16 bits per sample. In the experiments the Shimmer 3 device is fixed to the club's shaft just below the grip as seen in Fig. 3 .

Sensor signals are synchronized and processed by the distributed LabVIEW ${ }^{\text {TM }}$ application running on the laptop and cRIO platform. After streamed sensor signals are aligned by their impact samples, they are segmented into separate swings, each containing 1500 samples, with impact sample at index 1000. At the sampling frequency of $500 \mathrm{~Hz}$ the duration of each swing is $3 \mathrm{~s}$. In the graphs presented in this paper only swing signal samples between indexes 250 and 1000 or $1.5 \mathrm{~s}$ time frame are plotted.
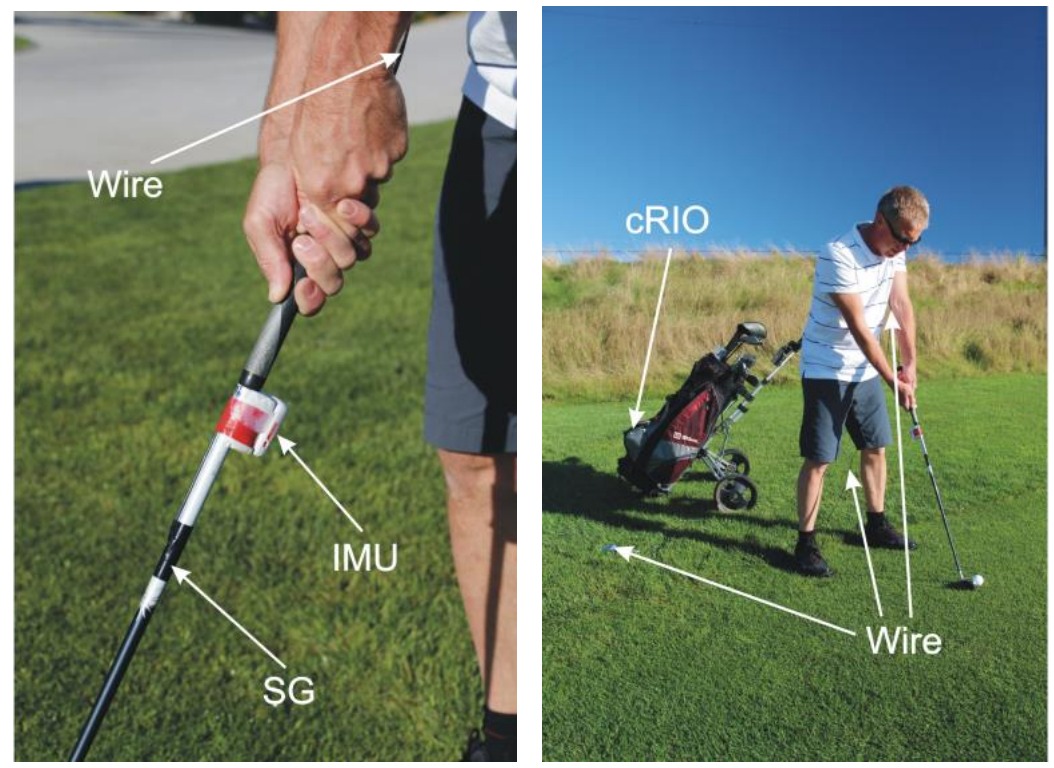

Fig. 3 Smart club prototype used in the field

Figure 4 shows the player signatures produced by the two strain gage sensors orthogonally placed on the shaft of the golf club. Fig. 4(a) shows trajectories (signatures) of a perfectly performed straight swing of three different players. It can be seen that their 
signatures are distinctively different. Figs. 4(b) and 4(c) show the trajectories of different swing types of player 1 and player 3 , respectively. It can be seen that the differences in trajectories of the same swing type of different players is greater than the difference in trajectories of the different swing types of the same player.

Figure 5 shows the sensor signals with marked points in time that correspond to the distinctive phases of the golf swing. Sensor signals are acquired by two strain gage sensors (top graph), 3-axis accelerometer (middle graph), and 3-axis gyroscope (bottom graph). Trajectories show high consistency of swings, repeatability, and precision of the measuring system.

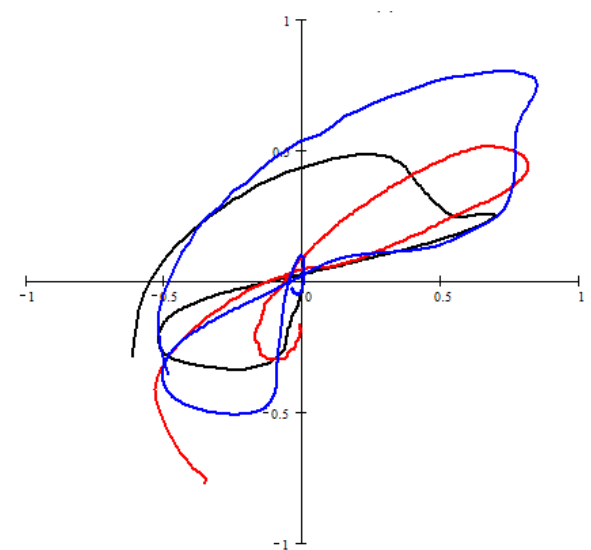

(a)

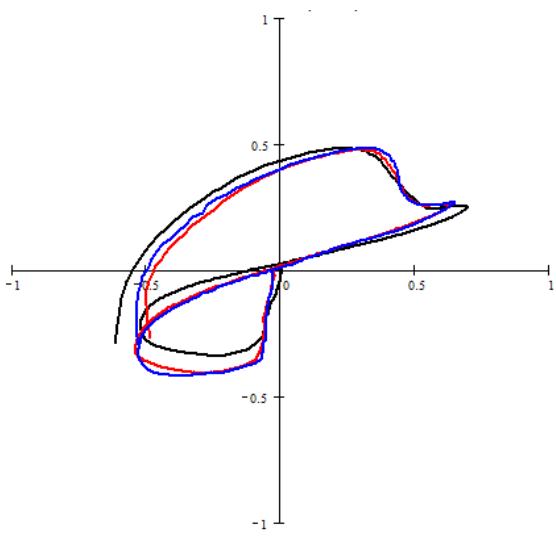

(b)

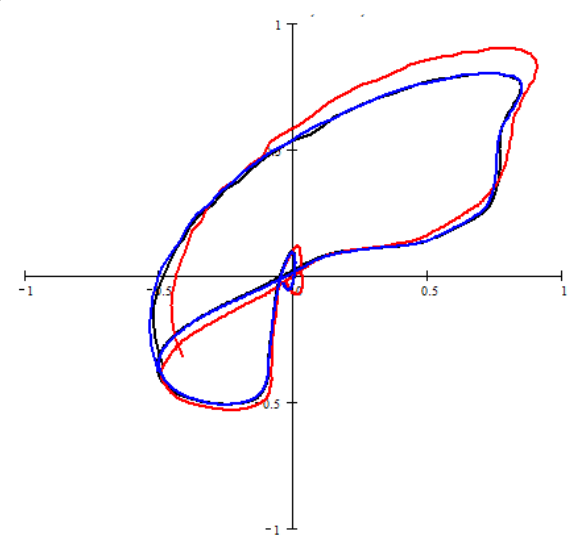

(c)

Fig. 4 Smart golf club prototype includes a 2D bending sensor; its trajectories confirm high consistency of players swings. Average trajectories $(\mathrm{N}=10)$ show (a) large differences between three different players' signatures, and much smaller differences between the perfect swing and faulty swings of the same player (b) and (c) 


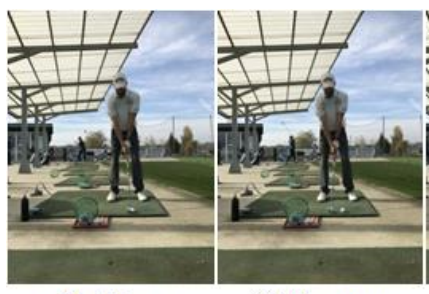

(a) address (b) takeaway

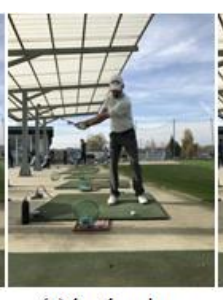

(c) backswing

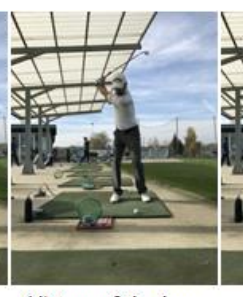

(d) top of the bsw

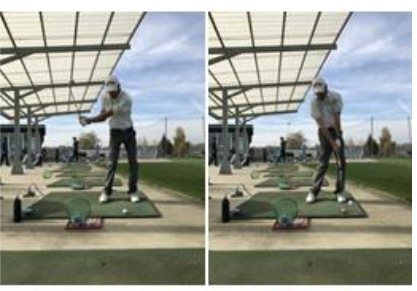

(e) downswing

(f) impact

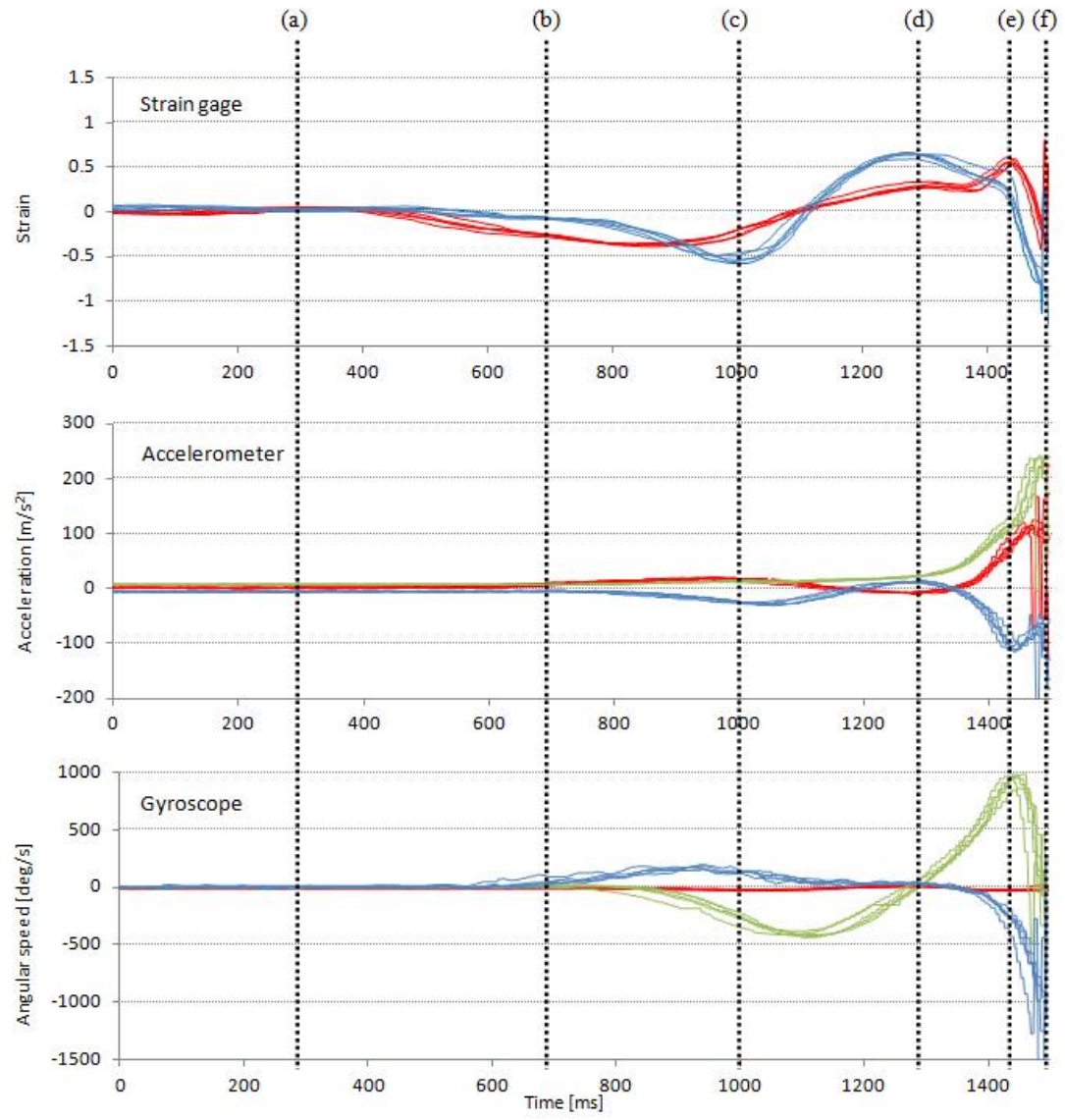

Fig. 5 Sensor signals and phases of the swing: (a) address, (b) takeaway, (c) backswing, (d) top of the backswing, (e) downswing, (f) impact. In the s train gage graph the red curves represent the response of the side-mounted sensor and the blue curves represent the response of the top-mounted sensor. In accelerometer graph red, blue, and green curves represent the acceleration in $1 x, 1 y$, and $1 z$ axis respectively. In the gyroscope graph red, blue, and green curves represent the angular velocity around $1 x, 1 y$, and $1 z$ axis, respectively. 


\subsection{Smart ski}

Smart ski prototype includes strain gage sensors for measuring the bend of the ski in several sections of the ski, several force sensors for measuring the force that the skier is applying to the ski, 3-axis accelerometer, and 3-axis gyroscope for measuring the motion. Bend and force sensors are integrated into the ski, accelerometer and gyroscope are attached to the skier's torso. Two additional Shimmer 3 devices are attached to the legs of the skier. The prototype is shown in Fig. 6; Smart skis are shown on the left hand side and the fully equipped skier to the right hand side of the figure.
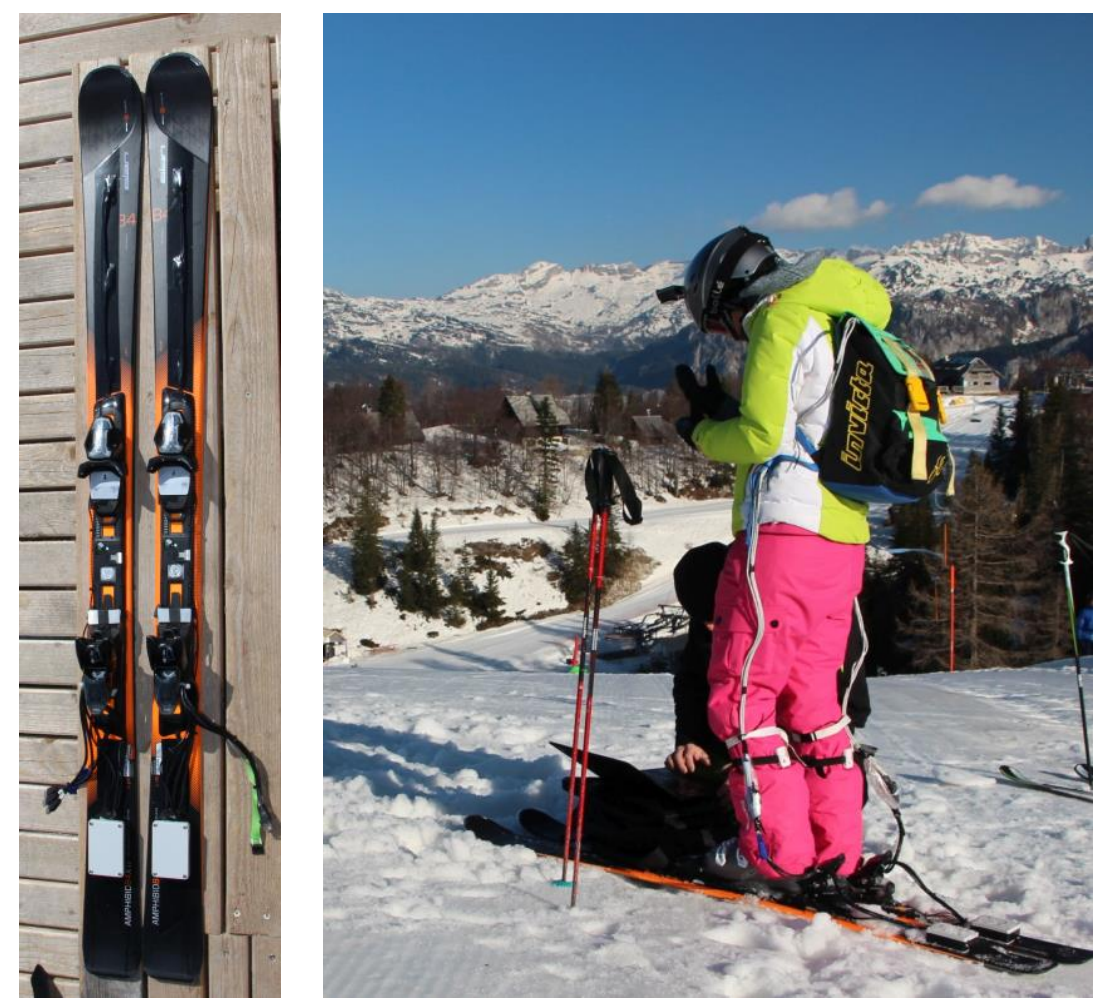

Fig. 6 Smart ski prototype

Sensor signals are collected, synchronized and processed by the LabVIEW ${ }^{\mathrm{TM}}$ application running on the professional measurement system (National Instruments Corporation, Austin, TX, USA) with NI cRIO 9063 base (667 MHz dual-core controller with FPGA) with bridge amplifier module NI 9237 and analogue to digital converter module NI 9205. The sampling frequency of the system is $100 \mathrm{~Hz}$. The accelerometer's dynamic range is up to $\pm 16 \mathrm{~g} 0$ and the gyroscopes dynamic range is up to $\pm 2000 \mathrm{deg} / \mathrm{s}$. The accelerometer and gyroscope are using the Wi-Fi connection (IEEE 802.11) to stream data to the cRIO device. Shimmer 3 devices are operating in the logging mode because of incompatibility of wireless technologies between the system elements; the laptop used in golf club prototype cannot be 
used during the skiing action. Sensor signals from Shimmer 3 device are synchronized with the rest of the sensors in post processing.

Laboratory tests for equipment operation testing, calibration and validation were followed by several snow tests in different weather and snow conditions and performed with different expert skiers, some ex world cup racers and some from the Slovenian Alpine Demo Team. Test skiers performed various skiing tasks and techniques according to the predefined schedule.

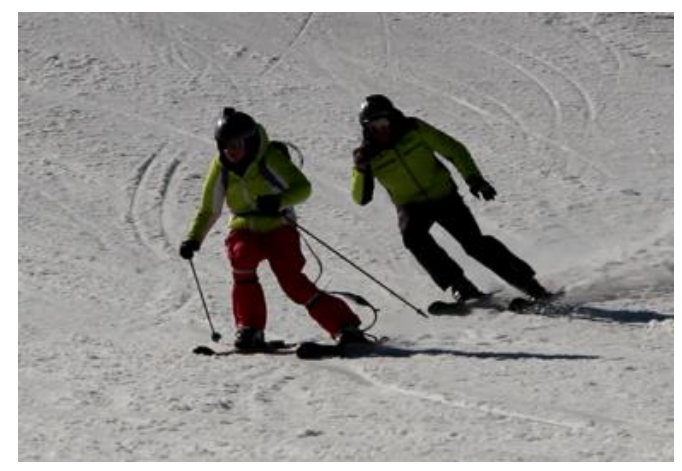

(a)

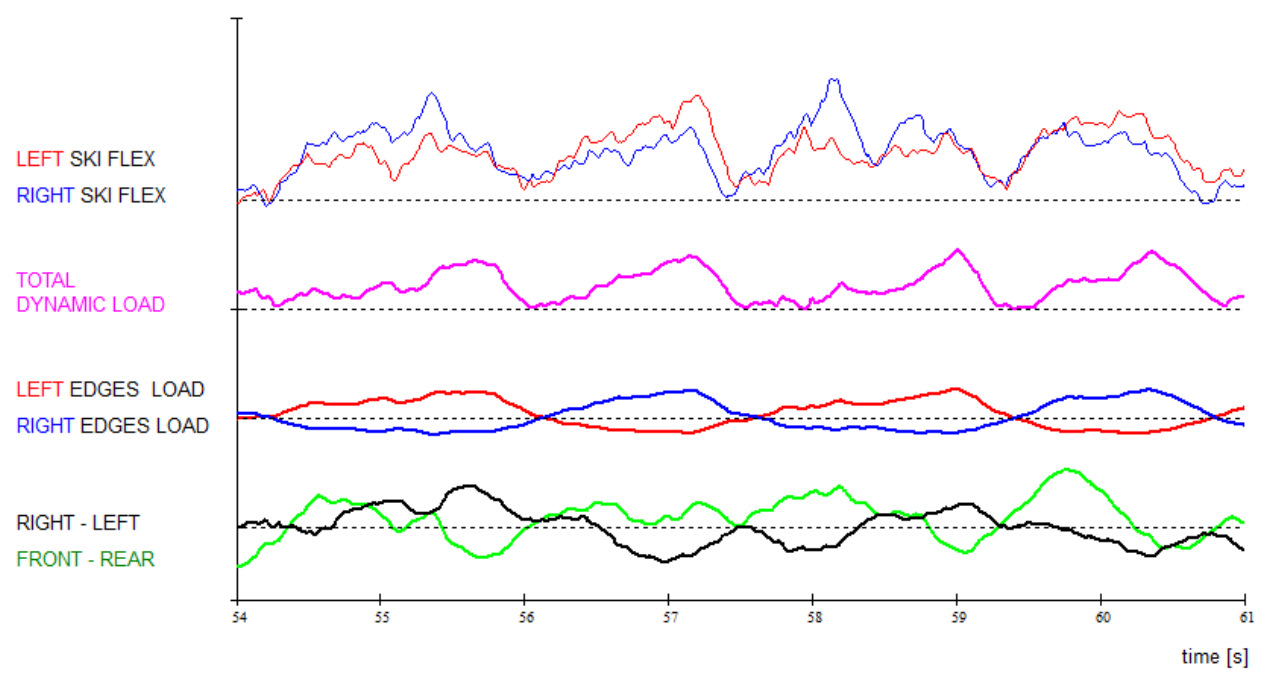

(b)

Fig. 7 Skiing experiments with smart ski prototype (a) and the corresponding signals and calculated plots (b) 
For example, one of the tasks was to perform the carving turns by equally loading both skis. Figure 7(a) shows the test skier during testing and Fig. 7(b) the corresponding signals acquired during the test. Figure 7(b), observing from top to bottom, is showing the following signals and calculated plots: a pair of signals shoving the flexing of the left and the right ski (narrow red and blue lines), total dynamic load applied to both skis (cyan line), a pair of plots showing the load on the left and the right ski edges (thick red and blue lines), relative load balance in the right-left direction (thick black line), and relative load balance in the front-rear direction (thick green line).

The final goal of the research is the development of the user application with real-time biofeedback. The feedback can be given through different modalities. An example of a real-time visual feedback, projected onto the skier's goggles, is shown in Fig. 8. The exemplary display includes the binary state indicators (carving) and sliders showing skiers current performance (outer/inner).

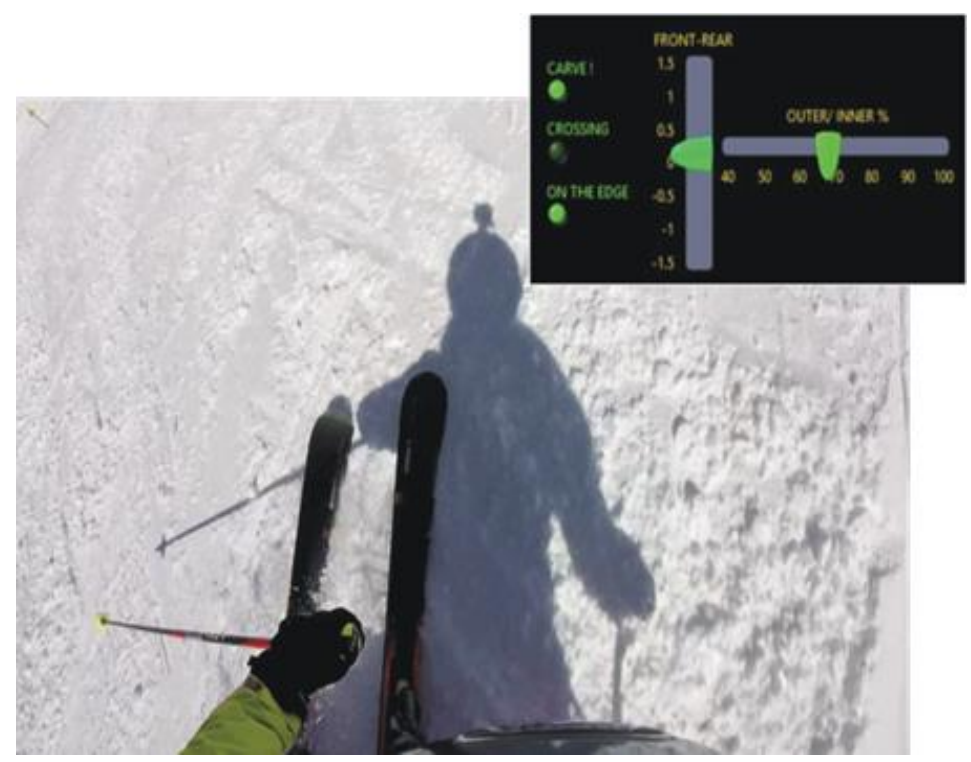

Fig. 8 Real-time biofeedback application

\section{CONCLUSION}

Biofeedback systems are important in motor learning in sports. Given the heterogeneity of sensors, actuators, and wireless technologies, countless scenarios of their use in biofeedback systems in sport are possible. Prototypes of a smart golf club and smart ski have been designed and the most important results of experimenting with both prototypes have been presented. With the smart golf club prototype it has been shown that the differences in trajectories of the same swing type of different players is greater than the difference in trajectories of the different swing types of the same player. The action of the skier and the reaction of the skis and terrain at the same time can be precisely and timely measured with the smart ski prototype. The acquired information from the integrated sensors is used in the 
testing of ski performance and for ski technique improvement or learning. The developed application allows the ski expert to analyze the performance of the skier based on several measured and calculated parameters. The application is currently capable of recognizing different phases of carving technique and diagnoses typical errors in regard to the load distribution during the steering phase of the turn.

The development of real-time biofeedback system usage concepts and procedures has been started based on the first measurement results. Concepts and procedures include a systematic approach by defining the useful short practice lessons, adapting the precision of real-time biofeedback system to the capabilities of the user (amateur vs. professional), the choice of correct feedback modality, and the appropriate amount of feedback information adapted to the limited perception capabilities of users during training.

With the development of real-time biofeedback systems we have been challenged by communication technologies limitations and limitations of the of-the-shelf sensor devices. It is important to accept that at this time none of the existing wireless technologies can satisfy all possible demands of different real-time biofeedback application scenarios. The main limitation here is the required real-time operation of the biofeedback system that at the same time requires a high bit rate, low delay, and long range. This can be a problem with highly dynamical human motion tracking and also with sport equipment that is usually used for enhancing or extending human motion, such as baseball bat, golf club, skis, ice hockey stick, and many others.

Acknowledgements: This work was supported in part by the Slovenian Research Agency within the research program Algorithms and Optimization Methods in Telecommunications.

\section{REFERENCES}

1. Bilodeau, E. A., Bilodeau, I. M., Alluisi, E.A., 1969, Principles of skill acquisition, Academic Press.

2. Sigrist, R., Rauter, G., Riener, R., Wolf, P., 2013, Augmented visual, auditory, haptic, and multimodal feedback in motor learning: a review, Psychonomic bulletin \& review, 20(1), pp. 21-53.

3. Giggins, O.M., Persson, U.M., Caulfield, B., 2013, Biofeedback in rehabilitation, Journal of neuroengineering and rehabilitation, $10(1), 60$, pp. 1-11.

4. Lightman, K., 2016, Silicon gets sporty, IEEE Spectrum, 53(3), pp. 48-53.

5. Nam, C.N.K., Kang, H.J., Suh, Y.S., 2014, Golf swing motion tracking using inertial sensors and a stereo camera, IEEE Transactions on Instrumentation and Measurement, 63(4), pp. 943-952.

6. Betzler, N.F., Monk, S.A., Wallace, E.S., Otto, S.R., 2012, Effects of golf shaft stiffness on strain, clubhead presentation and wrist kinematics, Sports biomechanics, 11(2), pp. 223-238.

7. Ueda, M., Negoro, H., Kurihara Y., Watanabe, K., 2013, Measurement of angular motion in golf swing by a local sensor at the grip end of a golf club, IEEE Transactions on Human-Machine Systems, 43(4), pp. 398-404.

8. Michahelles, F., Schiele, B., 2005, Sensing and monitoring professional skiers, IEEE Pervasive Computing, 4(3), pp. 40-45.

9. Kirby, R., 2009, Development of a real-time performance measurement and feedback system for alpine skiers, Sports Technology, 2(1-2), pp. 43-52.

10. Nakazato, K., Scheiber, P., Müller, E., 2011, A comparison of ground reaction forces determined by portable force-plate and pressure-insole systems in alpine skiing, J Sports Sci Med, 10(4), pp. 754-762.

11. Nemec, B., Petrič, T., Babič, J., Supej, M., 2014, Estimation of alpine skier posture using machine learning techniques, Sensors, 14(10), pp. 18898-18914.

12. Yu, G., Jang, Y.J., Kim, J., Kim, J.H., Kim, H.Y., Kim, K., Panday, S.B., 2016, Potential of IMU sensors in performance analysis of professional alpine skiers, Sensors, 16(4), 463.

13. Umek, A., Tomažič, S., Kos, A, 2015, Wearable training system with real-time biofeedback and gesture user interface, Personal and Ubiquitous Computing, 19(7), pp. 989-998. 
14. Kos, A., Tomažič, S., Umek, A., 2016, Suitability of smartphone inertial sensors for real-time biofeedback applications, Sensors, 16(3), 301.

15. Kos, A., Tomažič, S., Umek, A., 2016, Evaluation of smartphone inertial sensor performance for crossplatform mobile applications, Sensors, 16(4), 477.

16. Umek, A., Kos, A., 2016, Validation of smartphone gyroscopes for mobile biofeedback applications, Personal and Ubiquitous Computing, 20(5), pp. 657-666.

17. Umek, A., Zhang, Y., Tomažič, S., Kos, A., 2017, Suitability of Strain Gage Sensors for Integration into Smart Sport Equipment: A Golf Club Example, Sensors, 17(4), 916.

18. Baca, A., Dabnichki, P., Heller, M., Kornfeind, P., 2009, Ubiquitous computing in sports: A review and analysis, Journal of Sports Sciences, 27(12), pp. 1335-1346.

19. Baca, A., Kornfeind, P., 2006, Rapid feedback systems for elite sports training, IEEE Pervasive Computing, 5(4), pp. 70-76.

20. Mooney, R., Corley, G., Godfrey, A., Quinlan, L.R., ÓLaighin, G., 2015, Inertial sensor technology for elite swimming performance analysis: A systematic review, Sensors, 16(1), 18.

21. Llosa, J., Vilajosana, I., Vilajosana, X., Navarro, N., Surinach, E., Marques, J.M., 2009, REMOTE, a wireless sensor network based system to monitor rowing performance, Sensors, 9(9), pp. 7069-7082.

22. Tessendorf, B., Gravenhorst, F., Arnrich, B., Tröster, G., 2011, An imu-based sensor network to continuously monitor rowing technique on the water, Proc. Seventh IEEE International Conference on Intelligent Sensors, Sensor Networks and Information Processing (ISSNIP), 2011, pp. 253-258

23. Sturm, D., Yousaf, K., Eriksson, M., 2010, A wireless, unobtrusive kayak sensor network enabling feedback solutions, Proc. 2010 IEEE International conference on Body sensor networks (BSN), pp. 159-163.

24. Wang, Z., Wang, J., Zhao, H., Yang, N., Fortino, G., 2016, CanoeSense: Monitoring canoe sprint motion using wearable sensors, Proc. 2016 IEEE International Conference on Systems, Man, and Cybernetics (SMC), pp. 000644-000649.

25. Konttinen, N., Mononen, K., Viitasalo, J., Mets, T., 2004, The effects of augmented auditory feedback on psychomotor skill learning in precision shooting, Journal of Sport and Exercise Psychology, 26(2), pp. 306-316.

26. Cavallari, R., Martelli, F., Rosini, R., Buratti, C., Verdone, R., 2014, A survey on wireless body area networks: technologies and design challenges, IEEE Communications Surveys \& Tutorials, 16(3), pp. 1635-1657.

27. Chen, M., Gonzalez, S., Vasilakos, A., Cao, H., Leung, V.C., 2011, Body area networks: A survey, Mobile networks and applications, 16(2), pp. 171-193.

28. Cao, H., Leung, V., Chow, C., Chan, H., 2009, Enabling technologies for wireless body area networks: A survey and outlook, IEEE Communications Magazine, 47(12), pp. 84-93. 